\title{
Face Song Player According to Facial Expressions
}

\author{
Samule Lee, Seong-Yoon Shin \\ School of Computer Information \& Communication Engineering, Kunsan National University, Republic of Korea
}

\begin{tabular}{l} 
Article Info \\
\hline Article history: \\
Received Aug 07, 2016 \\
Revised Nov 10, 2016 \\
Accepted Nov 24, 2016 \\
\hline
\end{tabular}

Keyword:

Face song player

Facial contour lines

Facial expression

Facial shape information

MUCT DB

\begin{abstract}
Contemporary people have highly insufficient time and means of relieving their stress. Provision of a program that can solve such stress in daily life would make one's life substantially more enjoyable. In this thesis, Face Song Player, which is a system that recognizes the facial expression of an individual and plays music that is appropriate for such person, is presented. It studies information on the facial contour lines and extracts an average, and acquires the facial shape information. MUCT DB was used as the DB for learning. For the recognition of facial expression, an algorithm was designed by using the differences in the characteristics of each of the expressions on the basis of expressionless images. Facial expression is extracted by acquiring information on the eyes, eyebrows, eyelids, mouth, lips and nasal cheeks for expressions of happiness, surprise and sorrow as well as absence of expression. There is an advantage of being able to obtain a substantial effect with very low cost through this system.
\end{abstract}

Copyright $@ 2016$ Institute of Advanced Engineering and Science. All rights reserved.

\section{Corresponding Author:}

Seong-Yoon Shin,

School of Computer Information \& Communication Engineering,

Kunsan National University,

558 Daehak-ro, Gunsan-si-54150, Republic of Korea.

Email: s3397220@kunsan.ac.kr

\section{INTRODUCTION}

It is highly unlikely that no one among those living in the modern era experiences no stress. Stress manifests because of the need to adapt to an extensive range of changes in a short period of time. Leisureliness is increasingly disappearing from our modern society and people are subjected to marked stresses due to numerous reasons including monetary issues, social structure and interpersonal relationships, etc.

It is the reality that contemporary people have lack of time and means of relieving stress arising from their daily lives. Accordingly, we need a program that can relieve the stress we encounter in our daily life. For example, we need a system that recognizes us as friends and reads books by discerning our mood. In addition, it is much more effective if psychological pacification effects can be achieved at low cost. Moreover, such method would be substantially more effective if it could be used for a diverse range of purposes including education or games for the users.

Not everybody experiences the same stress from the same event. In fact, an appropriate level of stress can become the motivation to accomplish goals, induces motivation and provides vitality to life by becoming the catalyst in life. However, excessive stress damages health by imparting a substantial adverse effect on the body and mind because how one discerns and acts towards how stress effects the health and happiness in one's life. Therefore, it would be possible for people to lead their lives in a more beneficial direction if they could understand the causes of stress and find appropriate means of relieving stress.

Production and perception music encourages a broad range of sensory, cognitive and emotional processes. Emotion is the core function of the joy of music. Emotion can be deemed the core function of the joy of music along with the marked and diversified emotional states reported continuously by people throughout the period of listening to music [1]. As such, music imparts a substantial influence on our 
emotions. Playing music in accordance with one's facial expression could be very interesting and beneficial from the perspective of a therapeutic effect. Such a sensible process is manifested not only in music but also within a monitor. Expanding the horizontal angle of view in the monitor is an important characteristic of integral imaging monitors [2]. This method expands the angle of view to be changed by the appropriate application of our conversion algorithm by increasing the density of the image element in a horizontal direction.

Recent research on facial recognition include: [3] that researched the multi-directional multi-level dual cross pattern for powerful facial recognition; [4] that presented mobile application and a system referred to as OpenFace, which is a widely used facial recognition library; and [5] that asserted that the purpose of the research is facial recognition and emphasized facial recognition in the collection of the tracked faces in each of the photographs or video.

Research on expression recognition also include: [6] on the challenge of recognition and analysis of the second facial expression with the system called FERA2015; [7] that pursued automatic facial expression recognition by using an excellent facial patch function; and [8] that pursued a study on discriminative shared Gaussian processes for multi-view and view-invariant facial expression recognition.

\section{FACIAL RECOGNITION AND EXPRESSION RECOGNITION}

The face is recognized by using an Active Shape Model[9]. Facial recognition, as illustrated in Figure 1, is executed by computing the average of the information on the facial contour lines through learning.

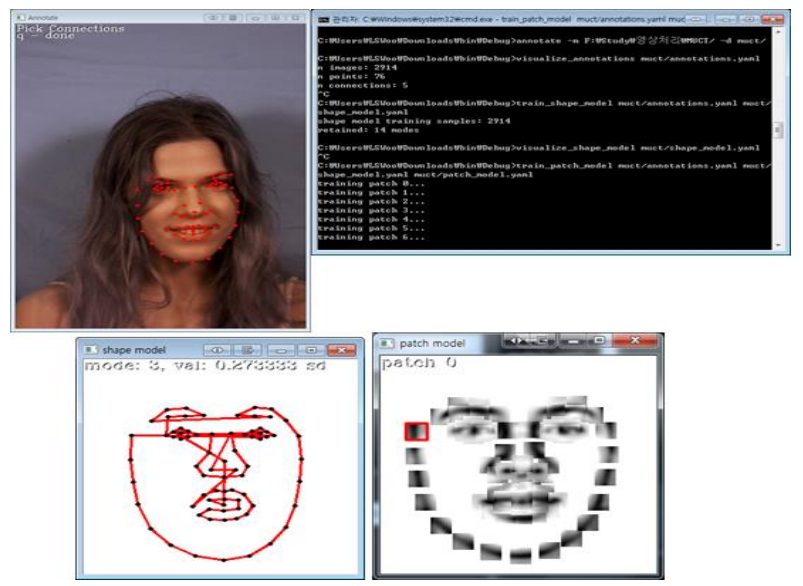

Figure 1. Face Recognition by Shape

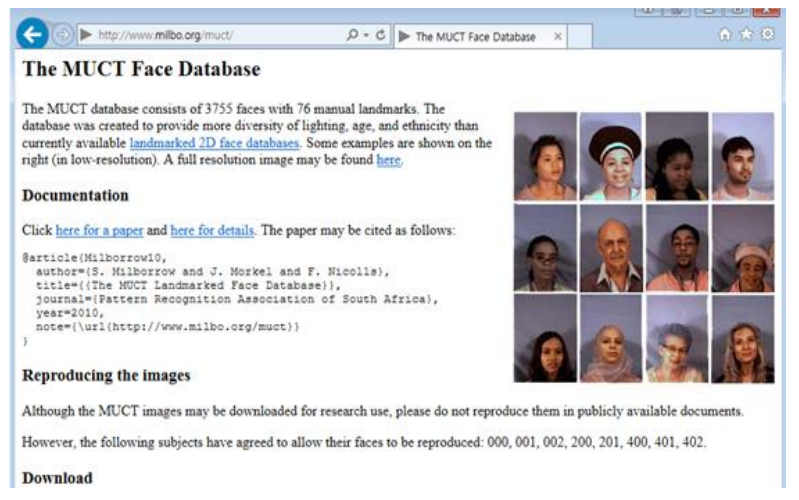

Figure 2. MUCT DB

MUCT DB [10] illustrated in Figure 2 was used as the learning data in this thesis. 
Expression recognition is executed in the following order. In the first several seconds, the data at the time of absence of expression is received, which is then used as the Standard to compute the distance between each of the characteristic points. Figure 3 is the data used in expression recognition.

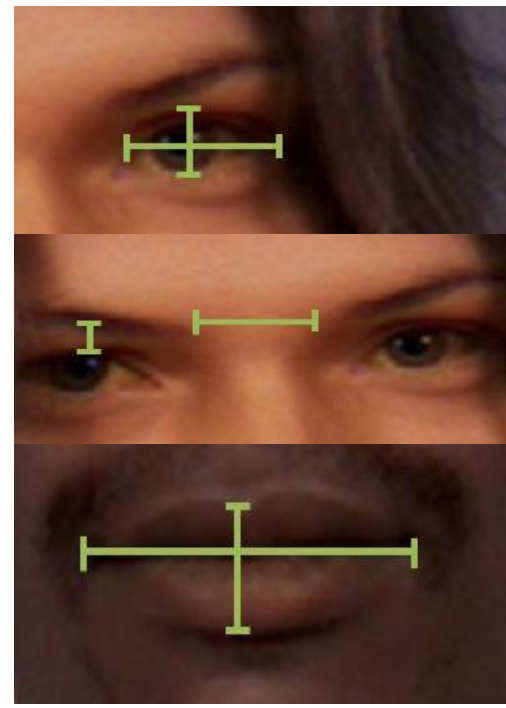

Figure 3. Data of Expression Recognition

(1) Happiness: Distance between philtrum and top of the center of the lips becomes closer. The distance between both the distal tip of the mouth increases.

Height of the eyes decreases. The both distal tips of the cheeks of the nose become broader.

Condition) Standard height of the eyes >Current height of the eyes

AND

Standard width of the mouth $<$ Current width of the mouth

(More than 5\%)

AND

Standard distance between the philtrum and lips>Current distance between the philtrum and lips

(More than 3\%)

\section{AND}

Standard distance between the both cheeks of the nose<Current distance between both cheeks of the nose

(More than 3\%)

(2) Sorrow: Width of the middle of the forehead becomes narrower, eyes get smaller and the distal tip of the mouth sags.

Conditions)Standard width of the middle of the forehead $>$ Width of the middle of the forehead

(More than 1\%)

\section{AND}

Standard height of the eyes $>$ Current height of the eyes

AND 
Standard distance between the chin and lower tip of the lips>Current between the philtrum and eyebrows

(More than 3\%)

(3) Surprise: Eyebrows are raised with the opening up of the mouth. The distance between the eyebrows and the top of the eyelids increases.

Conditions) Standard distance between the philtrum and eyebrows $<$ Current distance between the philtrum and eyebrows

(More than 3\%)

AND

Standard height of the mouth $>$ Current height of the mouth

AND

Standard distance between the eyebrows and the top of the eyelids $<$ Current distance between the eyebrows and the top of the eyelids

(More than 3\%)

\section{EXPERIMENT}

The experiment was realized by using Visual C++ 2013, and ASM recognition and MUCT DB were used together. Table 1 below illustrates the ASM matching recognition rates.

Table 1. ASM Recognition Ratio

\begin{tabular}{cccc}
\hline No. of faces & $\begin{array}{c}\text { No. of ASM } \\
\text { recognition }\end{array}$ & No. of errors & Recognition rate \\
\hline 40 & 34 & 6 & $85 \%$ \\
\hline
\end{tabular}

ASM matching errors occur due to the following reasons:

a. Wearing eye glasses with a thick frame can be recognized as eyes.

b. Not possible to recognize the face if the head is severely tilted or turned away.

c. Problem of recognizing creases in garments, etc. as the face.

d. Recognition of an error if a difference in light and shade occurs due to lighting.

e. Recognition of other objects if the background is complex.

Table 2 below illustrates the recognition rate of each of the expressions. Although the recognition rates for happiness, surprise and expressionless were high, that for a sorrowful expression was found to be low. This is a problem that occurs due to the lack of distinctness of the characteristics of a sorrowful expression in comparison to other expressions. In addition, there is the issue that the expression of sorrow differs between individuals.

Table 2. Expression Recognition Ratio

\begin{tabular}{ccccc}
\hline Categories & Expressionless(10) & Happiness(10) & Surprise(10) & Sorrow(10) \\
\hline $\begin{array}{c}\text { No. of } \\
\text { recognitions }\end{array}$ & 10 & 8 & 9 & 6 \\
No. of errors & 9 & 2 & 1 & 4 \\
Recognition rate & $90 \%$ & $80 \%$ & $90 \%$ & $60 \%$ \\
\hline
\end{tabular}

\section{CONCLUSION}

In this thesis, Face Song Player that recognizes the facial expression of people and playing music that is appropriate for the ambience of such expression was presented. An average was extracted through learning information on facial contour lines and the acquired information on facial shapes. MUCT DB was used as the DB for this purpose. For the recognition of the facial expressions, an algorithm was designed by using the differences in the special features of the expressions. Accordingly, facial expressions were 
extracted by acquiring information on the eyes, eyelids, eyebrows, mouth, lips and cheeks of the nose for happy, surprised, sorrowful and expressionless faces.

\section{REFERENCES}

[1] Patrik Vuilleumier and Wiebke Trost, "Music and emotions: from enchantment to entrainment", Ann. N.Y. Acad. Sci. Vol. 133, pp. 212-222, 2015.

[2] Adrián Dorado, Genaro Saavedra, Jorge Sola-Pikabea, and Manuel Martínez-Corral, "Integral Imaging Monitors with an Enlarged Viewing Angle", J. lnf. Commun. Converg. Eng. Vol. 13, No. 2, pp. 132-138, Jun. 2015.

[3] Ding, C., Choi, J., Tao, D., \& Davis, L.S., "Multi-directional multi-level dual-cross patterns for robust face recognition", IEEE transactions on pattern analysis and machine intelligence, 38(3), 518-531, 2016.

[4] Amos, B., Ludwiczuk, B., \& Satyanarayanan, M., "Open Face: A general-purpose face recognition library with mobile applications", 2016.

[5] Parkhi, Omkar M., Andrea Vedaldi, and Andrew Zisserman. "Deep face recognition", British Machine Vision Conference. Vol. 1. No. 3. 2015.

[6] Valstar, M.F., Almaev, T., Girard, J.M., Mc Keown, G., Mehu, M., Yin, L. \& Cohn, J.F., In Automatic Face and Gesture Recognition (FG)", Fera 2015-second facial expression recognition and analysis challenge, 2015 11th IEEE International Conference and Workshops on, Vol. 6, pp. 1-8, 2015, May.

[7] Happy, S.L., \& Routray, A., "Automatic facial expression recognition using features of salient facial patches", IEEE transactions on Affective Computing, 6(1), 1-12, 2015.

[8] Eleftheriadis, Stefanos, Ognjen Rudovic, and Maja Pantic. "Discriminative shared gaussian processes for multiview and view-invariant facial expression recognition", IEEE transactions on image processing 24.1, pp. 189-204, 2015.

[9] Tim Cootes, "An Introduction to Active Shape Models", Chapter 7: "Model-Based Methods in Analysis of Biomedical Images", in Image Processing and Analysis, Ed.R.Baldock and J.Graham, Oxford University Press, pp223-248, 2000

[10] Milborrow, S., Morkel, J., Nicolls, F. "The MUCT Landmarked Face Database”, Pattern Recognition Association of South Africa, 2010

\section{BIOGRAPHIES OF AUTHORS}

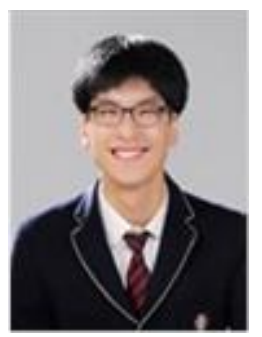

Samule Lee. He is currently attending fourth grade in School of Computer and Information Communication Engineering, Kunsan National University

His Interested Area : artificial intelligence, machine learning, computer vision

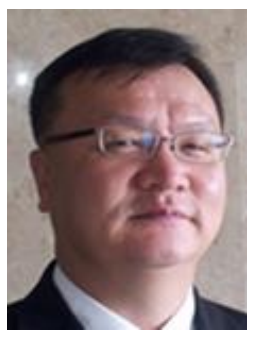

Seong-Yoon Shin. received his M.S. and Ph.D degrees from the Dept. of Computer Information Engineering of Kunsan National University, Kunsan, Korea, in 1997 and 2003, respectively. From 2006 to the present, he has been a professor in the School of Coputer Information \& Communication Engiineering. His research interests include Image Processing, Computer Vision, and Multimedia. 